\title{
T-Stability of Picard Iteration in Metric Spaces
}

\author{
Yuan Qing ${ }^{1}$ and B. E. Rhoades ${ }^{2}$ \\ ${ }^{1}$ Department of Mathematics, Beijing University of Aeronautics and Astronautics, \\ Beijing 100083, China \\ ${ }^{2}$ Department of Mathematics, Indiana University, Bloomington, IN 47405-7106, USA
}

Correspondence should be addressed to Yuan Qing, yuanqingbuaa@hotmail.com

Received 10 July 2007; Accepted 11 January 2008

Recommended by Hélène Frankowska

We establish a general result for the stability of Picard's iteration. Several theorems in the literature are obtained as special cases.

Copyright (C) 2008 Y. Qing and B. E. Rhoades. This is an open access article distributed under the Creative Commons Attribution License, which permits unrestricted use, distribution, and reproduction in any medium, provided the original work is properly cited.

Let $(X, d)$ be a complete metric space and $T$ a self-map of $X$. Let $x_{n+1}=f\left(T, x_{n}\right)$ be some iteration procedure. Suppose that $F(T)$, the fixed point set of $T$, is nonempty and that $x_{n}$ converges to a point $q \in F(T)$. Let $\left\{y_{n}\right\} \subset X$ and define $\epsilon_{n}=d\left(y_{n+1}, f\left(T, y_{n}\right)\right)$. If $\lim \epsilon_{n}=0$ implies that $\lim y_{n}=q$, then the iteration procedure $x_{n+1}=f\left(T, x_{n}\right)$ is said to be $T$-stable. Without loss of generality, we may assume that $\left\{y_{n}\right\}$ is bounded, for if $\left\{y_{n}\right\}$ is not bounded, then it cannot possibly converge. If these conditions hold for $x_{n+1}=T x_{n}$, that is, Picard's iteration, then we will say that Picard's iteration is $T$-stable.

We will obtain sufficient conditions that Picard's iteration is T-stable for an arbitrary self-map, and then demonstrate that a number of contractive conditions are Picard $T$-stable.

We will need the following lemma from [1].

Lemma 1. Let $\left\{x_{n}\right\},\left\{\epsilon_{n}\right\}$ be nonnegative sequences satisfying $x_{n+1} \leq h x_{n}+\epsilon_{n}$, for all $n \in \mathbb{N}, 0 \leq h<$ $1, \lim \epsilon_{n}=0$. Then, $\lim x_{n}=0$.

Theorem 1. Let $(X, d)$ be a nonempty complete metric space and $T$ a self-map of $X$ with $F(T) \neq \varnothing$. If there exist numbers $L \geq 0,0 \leq h<1$, such that

$$
d(T x, q) \leq L d(x, T x)+h d(x, q)
$$


for each $x \in X, q \in F(T)$, and, in addition,

$$
\lim d\left(y_{n}, T y_{n}\right)=0
$$

then Picard's iteration is T-stable.

Proof. First, we show that the fixed point $q$ of $T$ is unique. Suppose $p$ is another fixed point of $T$, then

$$
d(p, q)=d(T p, q) \leq L d(p, T p)+h d(p, q)=h d(p, q)
$$

Since $0 \leq h<1$, so $d(p, q)=0$, that is, $p=q$.

Let $\left\{y_{n}\right\} \subset X, \epsilon_{n}=d\left(y_{n+1}, T y_{n}\right)$, and $\lim \epsilon_{n}=0$. We need to show that $\lim y_{n}=q$.

Using (1), (2), and Lemma 1,

$$
d\left(y_{n+1}, q\right) \leq d\left(y_{n+1}, T y_{n}\right)+d\left(T y_{n}, q\right) \leq \epsilon_{n}+L d\left(y_{n}, T y_{n}\right)+h d\left(y_{n}, q\right)
$$

and $\lim y_{n}=q$.

Corollary 1. Let $(X, d)$ be a nonempty complete metric space and $T$ a self-map of $X$ satisfying the following: there exists $0 \leq h<1$, such that, for each $x, y \in X$,

$$
d(T x, T y) \leq h \max \{d(x, y), d(x, T x), d(y, T y), d(x, T y), d(y, T x)\}
$$

Then, Picard's iteration is T-stable.

Proof. From [2, Theorem 11], $T$ has a unique fixed point $q$. Also, $T$ satisfies (1). It remains to show that (2) is satisfied.

Define $p_{n}$ to be the diameter of the orbit of $y_{n}$; that is, $p_{n}=\delta\left(O\left(y_{n}, T y_{n}, \ldots\right)\right)$. First, we show that $p_{n}$ is bounded:

$$
\begin{aligned}
d\left(T y_{n}, q\right) & \leq h \max \left\{d\left(y_{n}, q\right), d\left(y_{n}, T y_{n}\right), d\left(y_{n}, T q\right), d\left(q, T y_{n}\right), d(q, T q)\right\} \\
& \leq h \max \left\{d\left(y_{n}, q\right), d\left(y_{n}, T y_{n}\right), d\left(y_{n}, q\right), d\left(q, T y_{n}\right), 0\right\} \\
& =h \max \left\{d\left(y_{n}, q\right), d\left(y_{n}, T y_{n}\right), d\left(y_{n}, q\right), d\left(q, T y_{n}\right)\right\}
\end{aligned}
$$

Hence, $d\left(T y_{n}, q\right) \leq h d\left(y_{n}, q\right)$ or $d\left(T y_{n}, q\right) \leq h d\left(y_{n}, T y_{n}\right)$ or $d\left(T y_{n}, q\right) \leq h d\left(q, T y_{n}\right)$.

If $d\left(T y_{n}, q\right) \leq h d\left(y_{n}, q\right)$, it is clear that

$$
d\left(T y_{n}, q\right) \leq h d\left(y_{n}, q\right) \leq \frac{h}{1-h} d\left(y_{n}, q\right)
$$

If $d\left(T y_{n}, q\right) \leq h d\left(q, T y_{n}\right)$, then

$$
d\left(T y_{n}, q\right)=0 \leq \frac{h}{1-h} d\left(y_{n}, q\right)
$$


If $d\left(T y_{n}, q\right) \leq h d\left(y_{n}, T y_{n}\right)$, then

$$
d\left(y_{n}, T y_{n}\right) \leq d\left(T y_{n}, q\right)+d\left(y_{n}, q\right) \leq h d\left(y_{n}, T y_{n}\right)+d\left(y_{n}, q\right)
$$

Hence, $d\left(T y_{n}, q\right) \leq(h /(1-h)) d\left(y_{n}, q\right)$. Now it is easy to see that $\left\{T y_{n}\right\}$ is bounded and so is $\left\{p_{n}\right\}$, since $\left\{y_{n}\right\}$ is bounded.

For any $i, j \geq n$, using (5),

$$
d\left(T y_{i}, T y_{j}\right) \leq h \max \left\{d\left(y_{i}, y_{j}\right), d\left(y_{i}, T y_{i}\right), d\left(y_{j}, T y_{j}\right), d\left(y_{i}, T y_{j}\right), d\left(y_{j}, T y_{i}\right)\right\} \leq h p_{n}
$$

Thus,

$$
d\left(y_{i}, T y_{j}\right) \leq d\left(y_{i}, T y_{i-1}\right)+d\left(T y_{i-1}, T y_{j}\right) \leq \epsilon_{i-1}+h p_{n-1}
$$

But

$$
d\left(y_{i}, y_{j}\right) \leq d\left(y_{i}, T y_{i-1}\right)+d\left(T y_{i-1}, T y_{j-1}\right)+d\left(T y_{j-1}, y_{j}\right) \leq \epsilon_{i-1}+h p_{n-1}+\epsilon_{i-1},
$$

which implies that

$$
p_{n} \leq 2 \epsilon_{i-1}+h p_{n-1}
$$

and $\lim p_{n}=0$ by Lemma 1 . Since $d\left(y_{n}, T y_{n}\right) \leq p_{n}, \lim d\left(y_{n}, T y_{n}\right)=0$.

The conclusion now follows from Theorem 1 .

Corollary 2 (see [3, Theorem 1]). Let $(X, d)$ be a nonempty complete metric space and $T$ a self-map of $X$ satisfying

$$
d(T x, T y) \leq L d(x, T x)+a d(x, y)
$$

for all $x, y \in X$, where $L \geq 0,0 \leq a<1$. Suppose that $T$ has a fixed point $p$. Then, $T$ is Picard $T$-stable.

Proof. Since $T$ satisfies (14) for all $x, y \in X$, then $T$ satisfies inequality (1) of our paper. Let $\left\{y_{n}\right\} \subset X$ and define $\epsilon_{n}=d\left(y_{n+1}, y_{n}\right)$. From the proof of Theorem 1 of [3], $\lim d\left(y_{n}, T y_{n}\right)=0$. Therefore, by our theorem (Theorem 1), $T$ is Picard $T$-stable.

Definition (5) of this paper is actually Definition (24) of [2]. Therefore, many contractive conditions are special cases of (5), and, for each of these, Picard's iteration is T-stable. For example, Theorems 1 and 2 of [4] and Theorem 1 of [5] are special cases of Corollary 1.

We will not examine the analogues of Theorem 1 for Mann, Ishikawa, Kirk, or any other iteration scheme since, if one obtains convergence to a fixed point for a map using Picard's iteration, there is no point in considering any other more complicated iteration procedure.

\section{Acknowledgment}

This article is partly supported by the National Natural Science Foundation of China (no. 10271012). 


\section{References}

[1] Q. Liu, "A convergence theorem of the sequence of Ishikawa iterates for quasi-contractive mappings," Journal of Mathematical Analysis and Applications, vol. 146, no. 2, pp. 301-305, 1990.

[2] B. E. Rhoades, "A comparison of various definitions of contractive mappings," Transactions of the American Mathematical Society, vol. 226, pp. 257-290, 1977.

[3] M. O. Osilike, "Stability results for fixed point iteration procedures," Journal of the Nigerian Mathematical Society, vol. 14-15, pp. 17-29, 1995.

[4] A. M. Harder and T. L. Hicks, "Stability results for fixed point iteration procedures," Mathematica Japonica, vol. 33, no. 5, pp. 693-706, 1988.

[5] B. E. Rhoades, "Fixed point theorems and stability results for fixed point iteration procedures," Indian Journal of Pure and Applied Mathematics, vol. 21, no. 1, pp. 1-9, 1990. 